\title{
Effects of environmental stresses and in vitro digestion on the release of tocotrienols encapsulated within chitosan-alginate microcapsules
}

\begin{abstract}
Considering the health benefits of tocotrienols, continuous works have been done on the encapsulation and delivery of these compounds. In this study, we encapsulated tocotrienols in chitosan-alginate microcapsules and evaluated their release profile. Generally, these tocotrienols microcapsules (TM) displayed high thermal stability. When subjected to $\mathrm{pH}$ adjustments ( $\mathrm{pH} 1-9)$, we observed that the release of tocotrienols was the highest (33.78 \pm $0.18 \%$ ) under basic conditions. The TM were also unstable against the effect of ionic strength, with a high release $(70.73 \pm 0.04 \%)$ of tocotrienols even at a low sodium chloride concentration $(50 \mathrm{mM})$. As for the individual isomers, $\delta$-tocotrienol was the most sensitive to $\mathrm{pH}$ and ionic strength. In contrast, $\beta$ - $/ \gamma$-tocotrienols were the most ionic-stable isomers but more responsive toward thermal treatment. Simulated gastrointestinal model showed that the chitosan-alginate-based TM could be used to retain tocotrienols in the gastric and subsequently release them in the intestines for possible absorption.
\end{abstract}

Keyword: Alginate; Chitosan; Gelation; Microencapsulation; Release profile; Selfassembled 